\title{
Hamming embedding and weak geometric consistency for large scale image search
}

\author{
Herve Jegou, Matthijs Douze, and Cordelia Schmid \\ INRIA Grenoble, LEAR, LJK \\ firstname.lastname@inria.fr
}

\begin{abstract}
This paper improves recent methods for large scale image search. State-of-the-art methods build on the bag-of-features image representation. We, first, analyze bag-of-features in the framework of approximate nearest neighbor search. This shows the suboptimality of such a representation for matching descriptors and leads us to derive a more precise representation based on 1) Hamming embedding (HE) and 2) weak geometric consistency constraints (WGC). HE provides binary signatures that refine the matching based on visual words. WGC filters matching descriptors that are not consistent in terms of angle and scale. $\mathrm{HE}$ and WGC are integrated within the inverted file and are efficiently exploited for all images, even in the case of very large datasets. Experiments performed on a dataset of one million of images show a significant improvement due to the binary signature and the weak geometric consistency constraints as well as their efficiency. Estimation of the full geometric transformation, i.e., a re-ranking step on a short list of images, is complementary to our weak geometric consistency constraints and allows to further improve the accuracy.
\end{abstract}

\section{Introduction}

We address the problem of searching for similar images in a large set of images. Similar images are defined as images of the same object or scene viewed under different imaging conditions, cf. Fig. 5 for examples. Many previous approaches have addressed the problem of matching such transformed images [1-5]. They are in most cases based on local invariant descriptors, and either match descriptors between individual images or search for similar descriptors in an efficient indexing structure. Various approximate nearest neighbor search algorithms such as kd-tree [1] or sparse coding with an overcomplete basis set [6] allow for fast search in small datasets. The problem with these approaches is that all individual descriptors need to be compared to and stored.

In order to deal with large image datasets, Sivic and Zisserman [4] introduced the bag-of-features (BOF) image representation in the context of image search. Descriptors are quantized into visual words with the $k$-means algorithm. An image is then represented by the frequency histogram of visual words obtained by assigning each descriptor of the image to the closest visual word. Fast access to 
the frequency vectors is obtained by an inverted file system. Note that this approach is an approximation to the direct matching of individual descriptors and somewhat decreases the performance. It compares favorably in terms of memory usage against other approximate nearest neighbor search algorithms, such as the popular Euclidean locality sensitive hashing (LSH) [7, 8]. LSH typically requires $100-500$ bytes per descriptor to index, which is not tractable, as a one million image dataset typically produces up to 2 billion local descriptors.

Some recent extensions of the BOF approach speed up the assignment of individual descriptors to visual words $[5,9]$ or the search for frequency vectors [10, 11]. Others improve the discriminative power of the visual words [12], in which case the entire dataset has to be known in advance. It is also possible to increase the performance by regularizing the neighborhood structure [10] or using multiple assignment of descriptors to visual words $[10,13]$ at the cost of reduced efficiency. Finally, post-processing with spatial verification, a re-occurring technique in computer vision [1], improves the retrieval performance. Such a postprocessing was recently evaluated in the context of large scale image search [9].

In this paper we present an approach complementary to those mentioned above. We make the distance between visual word frequency vectors more significant by using a more informative representation. Firstly, we apply a Hamming embedding (HE) to the descriptors by adding binary signatures which refine the visual words. Secondly, we integrate weak geometric consistency (WGC) within the inverted file system which penalizes the descriptors that are not consistent in terms of angle and scale. We also use a-priori knowledge on the transformations for further verification.

This paper is organized as follows. The interpretation of a BOF representation as a an image voting system is given in Section 2. Our contributions, HE and WGC, are described in sections 3 and 4. Complexity issues of our approach in the context of an inverted file system are discussed in Section 5. Finally, Section 6 presents the experimental results.

\section{Voting interpretation of bag-of-features}

In this section, we show how image search based on BOF can be interpreted as a voting system which matches individual descriptors with an approximate nearest neighbor (NN) search. We then evaluate BOF from this perspective.

\section{$2.1 \quad$ Voting approach}

Given a query image represented by its local descriptors $y_{i^{\prime}}$ and a set of database images $j, 1 \leq i \leq n$, represented by its local descriptors $x_{i, j}$, a voting system can be summarized as follows:

1. Dataset images scores $s_{j}$ are initialized to 0 .

2. For each query image descriptor $y_{i^{\prime}}$ and for each descriptor $x_{i, j}$ of the dataset, increase the score $s_{j}$ of the corresponding image by

$$
s_{j}:=s_{j}+f\left(x_{i, j}, y_{i^{\prime}}\right),
$$


where $f$ is a matching function that reflects the similarity between descriptors $x_{i, j}$ and $y_{i^{\prime}}$. For a matching system based on $\varepsilon$-search or $k-\mathrm{NN}, f(.,$. is defined as

$$
f_{\varepsilon}(x, y)=\left\{\begin{array}{ll}
1 & \text { if } d(x, y)<\varepsilon \\
0 & \text { otherwise }
\end{array} \quad f_{k-\mathrm{NN}}(x, y)= \begin{cases}1 & \text { if } x \text { is a } k \text {-NN of } y \\
0 & \text { otherwise }\end{cases}\right.
$$

where $d(.,$.$) is a distance (or dissimilarity measure) defined on the descriptor$ space. SIFT descriptors are typically compared using the Euclidean distance.

3. The image score $s_{j}^{*}=g_{j}\left(s_{j}\right)$ used for ranking is obtained from the final $s_{j}$. It can formally be written as

$$
s_{j}^{*}=g_{j}\left(\sum_{i^{\prime}=1 . . m^{\prime}} \sum_{i=1 . . . m_{j}} f\left(x_{i, j}, y_{i^{\prime}}\right)\right) .
$$

The simplest choice is $s_{j}^{*}=s_{j}$. In this case the score reflects the number of matches between the query and each database image. Note that this score counts possible multiple matches of a descriptor. Another popular choice is to take into account the number of image descriptors, for example $s_{j}^{*}=s_{j} / m_{j}$. The score then reflects the rate of descriptors that match.

\subsection{Bag-of-features: voting and approximate NN interpretation}

Bag-of-features (BOF) image search uses descriptor quantization. A quantizer $q$ is formally a function

$$
\begin{aligned}
q: \mathbb{R}^{d} & \rightarrow[1, k] \\
x & \mapsto q(x)
\end{aligned}
$$

that maps a descriptor $x \in \mathbb{R}^{d}$ to an integer index. The quantizer $q$ is often obtained by performing $k$-means clustering on a learning set. The resulting centroids are also referred to as visual words. The quantizer $q(x)$ is then the index of the centroid closest to the descriptor $x$. Intuitively, two descriptors $x$ and $y$ which are close in descriptor space satisfy $q(x)=q(y)$ with a high probability. The matching function $f_{q}$ defined as

$$
f_{q}(x, y)=\delta_{q(x), q(y)},
$$

allows the efficient comparison of the descriptors based on their quantized index. Injecting this matching function in (3) and normalizing the score by the number of descriptors of both the query image and the dataset image $j$, we obtain

$$
s_{j}^{*}=\frac{1}{m_{j} m^{\prime}} \sum_{i^{\prime}=1 . . m^{\prime}} \sum_{i=1 . . m_{j}} \delta_{q\left(x_{i, j}\right), q\left(y_{i^{\prime}}\right)}=\sum_{l=1 . . k} \frac{m_{l}^{\prime}}{m^{\prime}} \frac{m_{l, j}}{m_{j}},
$$

where $m_{l}^{\prime}$ and $m_{l, j}$ denote the numbers of descriptors, for the query and the dataset image $j$, respectively, that are assigned to the visual word $l$. Note that 
these scores correspond to the inner product between two BOF vectors. They are computed very efficiently using an inverted file, which exploits the sparsity of the BOF, i.e., the fact that $\delta_{q\left(x_{i, j}\right), q\left(y_{i^{\prime}}\right)}=0$ for most of the $\left(i, j, i^{\prime}\right)$ tuples.

At this point, these scores do not take into account the tf-idf weighting scheme (see [4] for details), which weights the visual words according to their frequency: rare visual words are assumed to be more discriminative and are assigned higher weights. In this case the matching function $f$ can be defined as

$$
f_{\text {tf-idf }}(x, y)=(\operatorname{tf-idf}(q(y)))^{2} \delta_{q(x), q(y)},
$$

such that the $t f-i d f$ weight associated with the visual word considered is applied to both the query and the dataset image in the BOF inner product. Using this new matching function, the image scores $s_{j}$ become identical to the BOF similarity measure used in [4]. This voting scheme normalizes the number of votes by the number of descriptors ( $L_{1}$ normalization). In what follows, we will use the $L_{2}$ normalization instead. For large vocabularies, the $L_{2}$ norm of a BOF is very close to the square root of the $L_{1}$ norm. In the context of a voting system, the division of the score by the $L_{2}$ norm is very similar to $s_{j}^{*}=s_{j} / \sqrt{m_{j}}$, which is a compromise between measuring the number and the rate of descriptor matches.

\subsection{Weakness of quantization-based approaches}

Image search based on BOF combines the advantages of local features and of efficient image comparison using inverted files. However, the quantizer reduces significantly the discriminative power of the local descriptors. Two descriptors are assumed to match if they are assigned the same quantization index, i.e., if they lie in the same Voronoi cell. Choosing the number of centroids $k$ is a compromise between the quantization noise and the descriptor noise.

Fig. 1(b) shows that a low value of $k$ leads to large Voronoi cells: the probability that a noisy version of a descriptor belongs to the correct cell is high. However, this also reduces the discriminative power of the descriptor: different descriptors lie in the same cell. Conversely, a high value of $k$ provides good precision for the descriptor, but the probability that a noisy version of the descriptor is assigned to the same cell is lower, as illustrated in Fig. 1(a).

We have measured the quality of the approximate nearest neighbor search performed by BOF in terms of the trade-off between 1) the average recall for the ground truth nearest neighbor and (b) the average rate of vectors that match in the dataset. Clearly, a good approximate nearest neighbor search algorithm is expected to make the nearest neighbor vote with high probability, and at the same time arbitrary vectors vote with low probability. In BOF, the tradeoff between these two quantities is managed by the number $k$ of clusters. For the evaluation, we have used the approximate nearest neighbor evaluation set available at [14]. It has been generated using the affine covariant features program of [15]. A one million vector set to be searched and a test query set of 10000 vectors are provided. All these vectors have been extracted from the INRIA Holidays image dataset described in Section 6. 


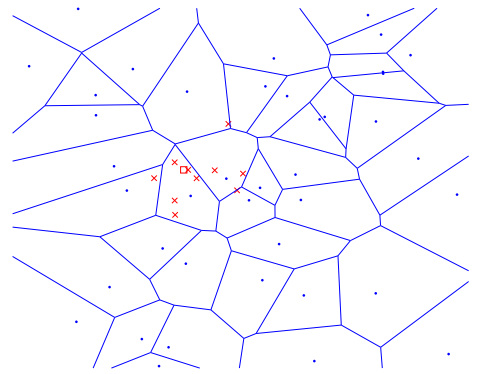

(a) $k=40$

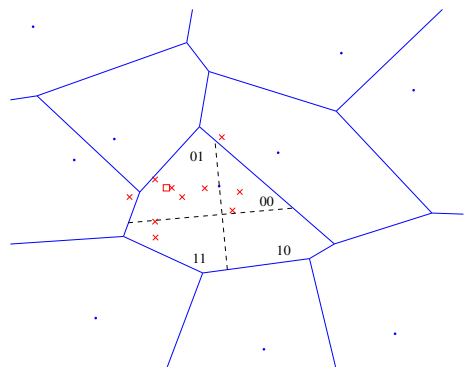

(b) $k=10$

Fig. 1. Illustration of $k$-means clustering and our binary signature. (a) Fine clustering. (b) Low $k$ and binary signature: the similarity search within a Voronoi cell is based on the Hamming distance. Legend: $\cdot=$ centroids, $\square=$ descriptor, $\times=$ noisy versions of the descriptor.

One can see in Fig. 2 that the performance of BOF as an approximate nearest neighbor search algorithm is of reasonable accuracy: for $k=1000$, the NN recall is of $45 \%$ and the proportion of the dataset points which are retrieved is of $0.1 \%$. One key advantage of BOF is that its memory usage is much lower than concurrent approximate nearest neighbor search algorithms. For instance, with 20 hash functions the memory usage of LSH [7] is of 160 bytes per descriptors compared to about 4 bytes for BOF. In next section, we will comment on the other curves of Fig. 2, which provide a much better performance than standard BOF.

\section{Hamming embedding of local image descriptors}

In this section, we present an approach which combines the advantages of a coarse quantizer (low number of centroids $k$ ) with those of a fine quantizer (high $k$ ). It consists in refining the quantized index $q\left(x_{i}\right)$ with a $d_{b}$-dimensional binary signature $b\left(x_{i}\right)=\left(b_{1}\left(x_{i}\right), \ldots, b_{d_{b}}\left(x_{i}\right)\right)$ that encodes the localization of the descriptor within the Voronoi cell, see Fig. 1(b). It is designed so that the Hamming distance

$$
h(b(x), b(y))=\sum_{1 \leq i \leq d_{b}} 1-\delta_{b_{i}(x), b_{i}(y)}
$$

between two descriptors $x$ and $y$ lying in the same cell reflects the Euclidean distance $d(x, y)$. The mapping from the Euclidean space into the Hamming space, referred to as Hamming Embedding (HE), should ensure that the Hamming distance $h$ between a descriptor and its NNs in the Euclidean space is small. 


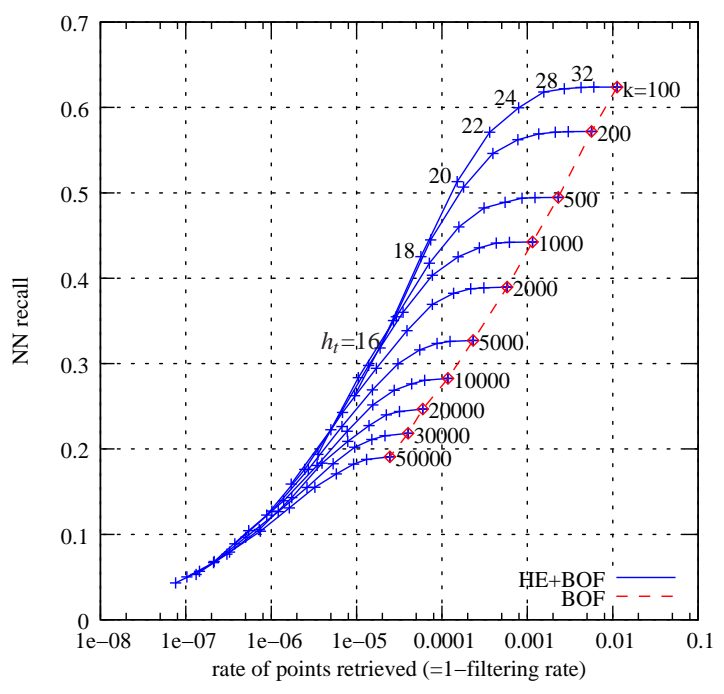

Fig. 2. Approximate nearest neighbor search accuracy of BOF (dashed) and Hamming Embedding (plain) for different numbers of clusters $k$ and Hamming thresholds $h_{t}$.

Note that this significantly different from the Euclidean version of LSH (E2LSH) $[7,8]$, which produces several hash keys per descriptor. In contrast, HE implicitly defines a single partitioning of the feature space and uses the Hamming metric between signatures in the embedded space.

We propose in the following a binary signature generation procedure. We distinguish between 1) the off-line learning procedure, which is performed on a learning dataset and generates a set of fixed values, and 2) the binary signature computation itself. The offline procedure is performed as follows:

1. Random matrix generation: $\mathrm{A} d_{b} \times d$ orthogonal projection matrix $P$ is generated. We randomly draw a matrix of Gaussian values and apply a $Q R$ factorization to it. The first $d_{b}$ rows of the orthogonal matrix obtained by this decomposition form the matrix $P$.

2. Descriptor projection and assignment: A large set of descriptors $x_{i}$ from an independent dataset is projected using $P$. These descriptors $\left(z_{i 1}, \ldots, z_{i d_{b}}\right)$ are assigned to their closest centroid $q\left(x_{i}\right)$.

3. Median values of projected descriptors: For each centroid $l$ and each projected component $h=1, \ldots, d_{b}$, we compute the median value $\tau_{l, h}$ of the set $\left\{z_{i h} \mid q\left(x_{i}\right)=l\right\}$ that corresponds to the descriptors assigned to the cell $l$.

The fixed projection matrix $P$ and $k \times d_{b}$ median values $\tau_{h, l}$ are used to perform the HE of a given descriptor $x$ by:

1. Assigning $x$ to its closest centroid, resulting in $q(x)$. 

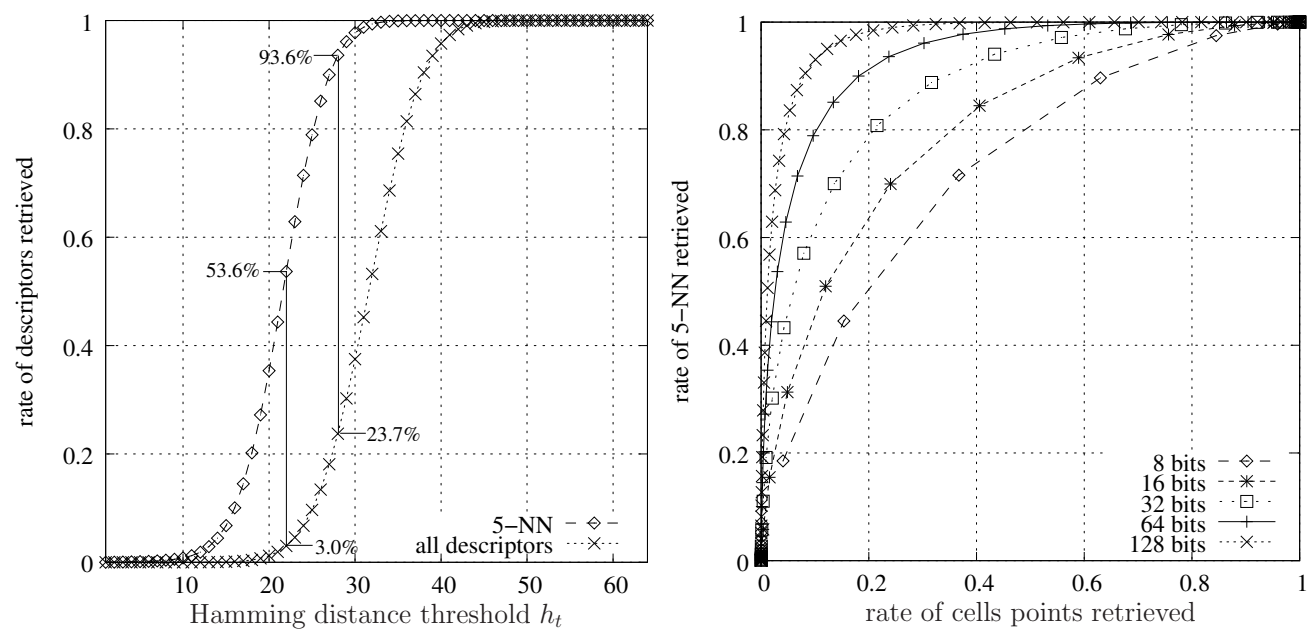

Fig. 3. Filtering effect of HE on the descriptors within a cell and on the $5 \mathrm{NNs}$. Left: trade-off between the rate of cell descriptors and the rate of $\mathrm{NN}$ that are retrieved for $d_{b}=64$. Right: impact of the number of bits $d_{b}$ of the binary signature length.

2. Projecting $x$ using $P$, which produces a vector $z=P x=\left(z_{1}, \ldots, z_{d_{b}}\right)$.

3. Computing the signature $b(x)=\left(b_{1}(x), \ldots, b_{d_{b}}(x)\right)$ as

$$
b_{i}(x)= \begin{cases}1 & \text { if } z_{i}>\tau_{q(x), i}, \\ 0 & \text { otherwise }\end{cases}
$$

At this point, a descriptor is represented by $q(x)$ and $b(x)$. We can now define the HE matching function as

$$
f_{\mathrm{HE}}(x, y)= \begin{cases}\operatorname{tf}-\operatorname{idf}(q(x)) & \text { if } q(x)=q(y) \text { and } h(b(x), b(y)) \leq h_{t} \\ 0 & \text { otherwise }\end{cases}
$$

where $h$ is the Hamming distance defined in Eqn. 9 and $h_{t}$ is a fixed Hamming threshold such that $0 \leq h_{t} \leq d_{b}$. It has to be sufficiently high to ensure that the Euclidean NNs of $x$ match, and sufficiently low to filter many points that lie in a distant region of the Voronoi cell. Fig. 3 depicts this compromise. The plots have been generated by analyzing a set of 1000 descriptors assigned to the same centroid. Given a descriptor $x$ we compare the rate of descriptors that are retrieved by the matching function to the rate of $5-\mathrm{NN}$ that are retrieved.

The left plot shows that the choice of an appropriate threshold $h_{t}$ (here between 20 and 30) ensures that most of the cell's descriptors are filtered and that the descriptor's NNs are preserved with a high probability. For instance, setting $h_{t}=22$ filters about $97 \%$ of the descriptors while preserving $53 \%$ of the 5 -NN. A higher value $h_{t}=28$ keeps $94 \%$ of the 5 -NN and filters $77 \%$ of the cell descriptors. Fig. 3(right) represents this trade-off for different binary signature lengths. Clearly, the longer the binary signature $d_{b}$, the better the HE filtering 
quality. In the following, we have fixed $d_{b}=64$, which is a good compromise between HE accuracy and memory usage ( 8 bytes per signature).

The comparison with standard BOF shows that the approximate nearest neighbor search performed by $\mathrm{BOF}+\mathrm{HE}$ is much better, see Fig. 2. Using HE for the same number of vectors that are retrieved, increases the probability that the $\mathrm{NN}$ is among these voting vectors.

\section{Large-scale geometric consistency}

BOF based image search ranks the database images without exploiting geometric information. Accuracy may be improved by adding a re-ranking stage [9] that computes a geometric transformation between the query and a shortlist of dataset images returned by the BOF search. To obtain an efficient and robust estimation of this transformation, the model is often kept as simple as possible $[1,9]$. In [1] an affine 2D transformation is estimated in two stages. First, a Hough scheme estimates a transformation with 4 degrees of freedom. Each pair of matching regions generates a set of parameters that "vote" in a 4D histogram. In the second stage, the sets of matches from the largest bins are used to estimate a finer 2D affine transform. In [9] further efficiency is obtained by a simplified parameter estimation and an approximate local descriptor matching scheme.

Despite these optimizations, existing geometric matching algorithms are costly and cannot reasonably be applied to more than a few hundred images. In this section, we propose to exploit weak, i.e., partial, geometrical information without explicitly estimating a transformation mapping the points from an image to another. The method is integrated into the inverted file and can efficiently be applied to all images. Our weak geometric consistency constraints refine the voting score and make the description more discriminant. Note that a re-ranking stage [9] can, in addition, be applied on a shortlist to estimate the full geometric transformation. It is complementary to the weak consistency constraints (see Section 6).

\subsection{Weak geometrical consistency}

The key idea of our method is to verify the consistency of the angle and scale parameters for the set of matching descriptors of a given image. We build upon and extend the BOF formalism of (1) by using several scores $s_{j}$ per image. For a given image $j$, the entity $s_{j}$ then represents the histogram of the angle and scale differences, obtained from angle and scale parameters of the interest regions of corresponding descriptors. Although these two parameters are not sufficient to map the points from one image to another, they can be used to improve the image ranking produced by the inverted file. This is obtained by modifying the update step of (1) as follows:

$$
s_{j}\left(\delta_{a}, \delta_{s}\right):=s_{j}\left(\delta_{a}, \delta_{s}\right)+f\left(x_{i, j}, y_{i^{\prime}}\right),
$$


where $\delta_{a}$ and $\delta_{s}$ are the quantized angle and $\log$-scale differences between the interest regions. The image score becomes

$$
s_{j}^{*}=g\left(\max _{\left(\delta_{a}, \delta_{s}\right)} s_{j}\left(\delta_{a}, \delta_{s}\right)\right) .
$$

The motivation behind the scores of (12) is to use angle and scale information to reduce the scores of the images for which the points are not transformed by consistent angles and scales. Conversely, a set of points consistently transformed will accumulate its votes in the same histogram bin, resulting in a high score.

Experimentally, the quantities $\delta_{a}$ and $\delta_{s}$ have the desirable property of being largely independent: computing separate histograms for angle and scale is as precise as computing the full 2D histogram of (11). In this case two histograms $s_{j}^{a}$ and $s_{j}^{s}$ are separately updated by

$$
\begin{gathered}
s_{j}^{a}\left(\delta_{a}\right):=s_{j}^{a}\left(\delta_{a}\right)+f\left(x_{i, j}, y_{i^{\prime}}\right) \\
s_{j}^{s}\left(\delta_{s}\right):=s_{j}^{s}\left(\delta_{s}\right)+f\left(x_{i, j}, y_{i^{\prime}}\right) .
\end{gathered}
$$

The two histograms can be seen as marginal probabilities of the $2 \mathrm{D}$ histogram. Therefore, the final score

$$
s_{j}^{*}=g\left(\min \left(\max _{\delta_{a}} s_{j}^{a}\left(\delta_{a}\right), \max _{\delta_{s}} s_{j}^{s}\left(\delta_{s}\right)\right)\right)
$$

is a reasonable estimate of the maximum of (12). This approximation will be used in the following. It significantly reduces the memory and CPU requirements. In practice, the histograms are smoothed by a moving average to reduce the angle and log-scale quantization artifacts. Note that the translation could be theoretically included in WGC. However, for a large number of images, the number of parameters should be in fewer than 2 dimensions, otherwise the memory and CPU costs of obtaining the scores would not be tractable.

\subsection{Injecting a priori knowledge}

We have experimentally observed that the repartition of the angle difference $\delta_{a}$ is different for matching and non-matching image pairs: the angle difference for the matching points follows a non-uniform repartition. This is due to the human tendency to shoot either in "portrait" or "landscape" mode. A similar bias is observed for $\delta_{s}$ : image pairs with the same scale $\left(\delta_{s}=0\right)$ are more frequent. We use the orientation and scale priors to weight the entries of our histograms before extracting their maxima. We have designed two different orientation priors: "same orientation" for image datasets known to be shot with the same orientation (i.e. Oxford) and " $\pi / 2$ rotation" for more general bases (i.e. Holidays). 


\section{Complexity}

Both HE and WGC are integrated in the inverted file. This structure is usually implemented as an array that associates a list of entries with each visual word. Each entry contains a database image identifier and the number of descriptors of this image assigned to this visual word. The tf-idf weights and the BOF vector norms can be stored separately. The search consists in iterating over the entries corresponding to the visual words in the query image and in updating the scores accordingly.

An alternative implementation consists in storing one entry per descriptor in the inverted list corresponding to a visual word instead of one entry per image. This is almost equivalent for very large vocabularies, because in this case multiple occurrences of a visual word on an image are rare, i.e., it is not necessary to store the number of occurrences. In our experiments, the overall memory usage was not noticeably changed by this implementation. This implementation is required by HE and WGC, because additional information is stored per local descriptor.

HE impact on the complexity: For each inverted file entry, we compute the Hamming distance between the signature of the query and that of the database entry. This is done efficiently with a binary xor operation. Entries with a distance above $h_{t}$ are rejected, which avoids the update of image scores for these entries. Note that this occurs for a fair rate of entries, as shown in Fig. 3.

WGC impact on the complexity: WGC modifies the score update by applying (13) instead of (1). Hence, two bins are updated, instead of one for a standard inverted file. The score aggregation as well as histogram smoothing have negligible computing costs. With the tested parameters, see Table 1(left), the memory usage of the histogram scores is 128 floating point values per image, which is small compared with the inverted lists.

Runtime: All experiments were carried out on $2.6 \mathrm{GHz}$ quad-core computers. As the new inverted file contains more information, we carefully designed the size of the entries to fit a maximum 12 bytes per point, as shown in Table 1(left).

Table 1(right) summarizes the average query time for a one million image dataset. We observe that the binary signature of HE has a negligible computational cost. Due to the high rate of zero components of the BOF for a visual vocabulary of $k=200000$, the search is faster. Surprisingly, HE reduces the inverted file query time. This is because the Hamming distance computation and thresholding is cheaper than updating the scores. WGC reduces the speed, mostly because the histograms do not fit in cache memory and their memory access pattern is almost random. Most interestingly the search time of $\mathrm{HE}+$ WGC is comparable to the inverted file baseline. Note that for $k=200000$ visual words, the assignment uses a fast approximate nearest neighbor search, i.e., the computation is not ten times slower than for $k=20000$, which here uses exhaustive search. 
Table 1. Inverted file memory usage and query time per image for a quad-core.

\begin{tabular}{|c|c|c|c|c|c|}
\hline \multirow{4}{*}{\multicolumn{2}{|c|}{$\begin{array}{l}\text { image id } \\
\text { orientation } \\
\text { log-scale } \\
\text { binary signature }\end{array}$}} & \multirow{4}{*}{$\begin{array}{r}21 \text { bits } \\
6 \text { bits } \\
5 \text { bits } \\
64 \text { bits }\end{array}$} & \multirow{3}{*}{$\begin{array}{l}\text { compute descriptors } \\
\text { quantization }+ \text { binary signature }\end{array}$} & \multirow{2}{*}{\multicolumn{2}{|c|}{$\frac{k=20000 \quad k=200000}{0.88 \mathrm{~s}}$}} \\
\hline & & & & & \\
\hline & & & & \multicolumn{2}{|l|}{$036 \mathrm{~s}$} \\
\hline & & & search, baseline & $2.74 \mathrm{~s}$ & $0.62 \mathrm{~s}$ \\
\hline \multirow{3}{*}{ total } & WGC & 4 bytes & search, WGC & $10.19 \mathrm{~s}$ & $2.11 \mathrm{~s}$ \\
\hline & $\mathrm{HE}$ & 12 bytes & search, HE & $1.16 \mathrm{~s}$ & $0.20 \mathrm{~s}$ \\
\hline & WGC+HE & 12 bytes & search, HE+WGC & $1.82 \mathrm{~s}$ & $0.65 \mathrm{~s}$ \\
\hline
\end{tabular}

\section{Experiments}

We perform our experiments on two annotated datasets: our own Holidays dataset, see Fig. 5, and the Oxford5k dataset. To evaluate large scale image search we also introduce a distractor dataset downloaded from Flickr. For evaluation we use mean average precision (mAP) [9], i.e., for each query image we obtain a precision/recall curve, compute its average precision and then take the mean value over the set of queries. Descriptors are obtained by the Hessian-Affine detector and the SIFT descriptor, using the software of [15] with the default parameters. Clustering is performed with $k$-means on the independent Flickr60k dataset. The number of clusters is specified for each experiment.

\subsection{Datasets}

In the following we present the different datasets used in our experiments.

Holidays (1491 images, 4.456M descriptors, 500 queries). We have collected a new dataset which mainly contains personal holiday photos. The remaining ones were taken on purpose to test the robustness to various transformations: rotations, viewpoint and illumination changes, blurring, etc. The dataset includes a very large variety of scene types (natural, man-made, water and fire effects, etc) and images are of high resolution. The dataset contains 500 image groups, each of which represents a distinct scene. The first image of each group is the query image and the correct retrieval results are the other images of the group. The dataset is available at [14].

Oxford5k (5062 images, 15.886M descriptors, 55 queries). We also used the Oxford dataset [9]. The images represent Oxford buildings. All the dataset images are in "upright" orientation because they are displayed on the web.

Flickr60k (67r14 images, $140 M$ descriptors) and Flickr1M (1M images, 2072M descriptors). We retrieved arbitrary images from Flickr and built two distinct sets: Flickr60k is used to learn the quantization centroids and the HE parameters (median values). For these tasks we have used respectively $5 \mathrm{M}$ and $140 \mathrm{M}$ descriptors. Flickr1M are distractor images for large scale image search. Compared to Holidays, the Flickr datasets are slightly biased, because they include low-resolution images and more photos of humans. 
Table 2. Results for Holidays and Oxford datasets. mAP scores for the baseline, HE, WGC and HE+WGC. Angle prior: same orientation for Oxford, $0, \pi / 2, \pi$ and $3 \pi / 2$ rotations for Holidays. Vocabularies are generated on the independent Flickr60K dataset.

\begin{tabular}{|c|c|c|c|c|c|c|}
\hline & \multicolumn{2}{|c|}{ Parameters } & \multirow{2}{*}{\multicolumn{2}{|c|}{$\begin{array}{c}\text { Holidays } \\
k=20000 k=200000\end{array}$}} & \multirow{2}{*}{\multicolumn{2}{|c|}{$\begin{array}{c}\text { Oxford } \\
k=20000 k=200000\end{array}$}} \\
\hline & $\mathrm{HE}: h_{t}$ & WGC & & & & \\
\hline baseline & & & 0.4463 & 0.5488 & 0.3854 & 0.3950 \\
\hline $\mathrm{HE}$ & 20 & & 0.7268 & 0.7093 & 0.4798 & 0.4503 \\
\hline $\mathrm{HE}$ & 22 & & 0.7181 & 0.7074 & 0.4892 & 0.4571 \\
\hline $\mathrm{HE}$ & 24 & & 0.6947 & 0.7115 & 0.4906 & 0.4585 \\
\hline $\mathrm{HE}$ & 26 & & 0.6649 & 0.6879 & 0.4794 & 0.4624 \\
\hline WGC & & no prior & 0.5996 & 0.6116 & 0.3749 & 0.3833 \\
\hline WGC & & with prior & 0.6446 & 0.6859 & 0.4375 & 0.4602 \\
\hline $\mathrm{HE}+\mathrm{WGC}$ & 20 & with prior & 0.7391 & 0.7328 & 0.5442 & 0.5096 \\
\hline $\mathrm{HE}+\mathrm{WGC}$ & 22 & with prior & 0.7463 & 0.7382 & 0.5472 & 0.5217 \\
\hline $\mathrm{HE}+\mathrm{WGC}$ & 24 & with prior & 0.7507 & 0.7439 & 0.5397 & 0.5252 \\
\hline $\mathrm{HE}+\mathrm{WGC}$ & 26 & with prior & 0.7383 & 0.7404 & 0.5253 & 0.5275 \\
\hline
\end{tabular}

Impact of the clustering learning set. Learning the visual vocabulary on a distinct dataset shows more accurately the behavior of the search in very large image datasets, for which 1) query descriptors represent a negligible part of the total number of descriptors, and 2) the number of visual words represents a negligible fraction of the total number of descriptors. This is confirmed by comparing our results on Oxford to the ones of [9], where clustering is performed on the evaluation set. In our case, i.e., for a distinct visual vocabulary, the improvement between a small and large $k$ is significantly reduced when compared to [9], see first row of Table 2.

\subsection{Evaluation of $\mathrm{HE}$ and WGC}

INRIA Holidays and Oxford building datasets: Table 2 compares the proposed methods with the standard BOF baseline. We can observe that both HE and WGC result in significant improvements. Most importantly, the combination of the two further increases the performance.

Large scale experiments: Fig. 4 shows an evaluation of the different approaches for large datasets, i.e., we combined the Holidays dataset with a varying number of images from the $1 \mathrm{M}$ Flickr dataset. We clearly see that the gain of the variant WGC + HE is very significant. In the case of WGC + HE the corresponding curves degrade less rapidly when the number of images in the database increases. Results for various queries are presented in Fig. 5. We can observe that $\mathrm{HE}$ and WGC improve the quality of the ranking significantly. Table 3 measures this improvement. It gives the rate of true positives that are in a shortlist of 100 images. For a dataset of one million images, the baseline only returns $31 \%$ of the true positive, against $62 \%$ for HE+WGC. This reflects the quality of the shortlist that will be considered in a re-ranking stage.

Re-ranking: The re-ranking is based on the estimation of an affine transformation with our implementation of [1]. Fig. 4 also shows the results obtained with 

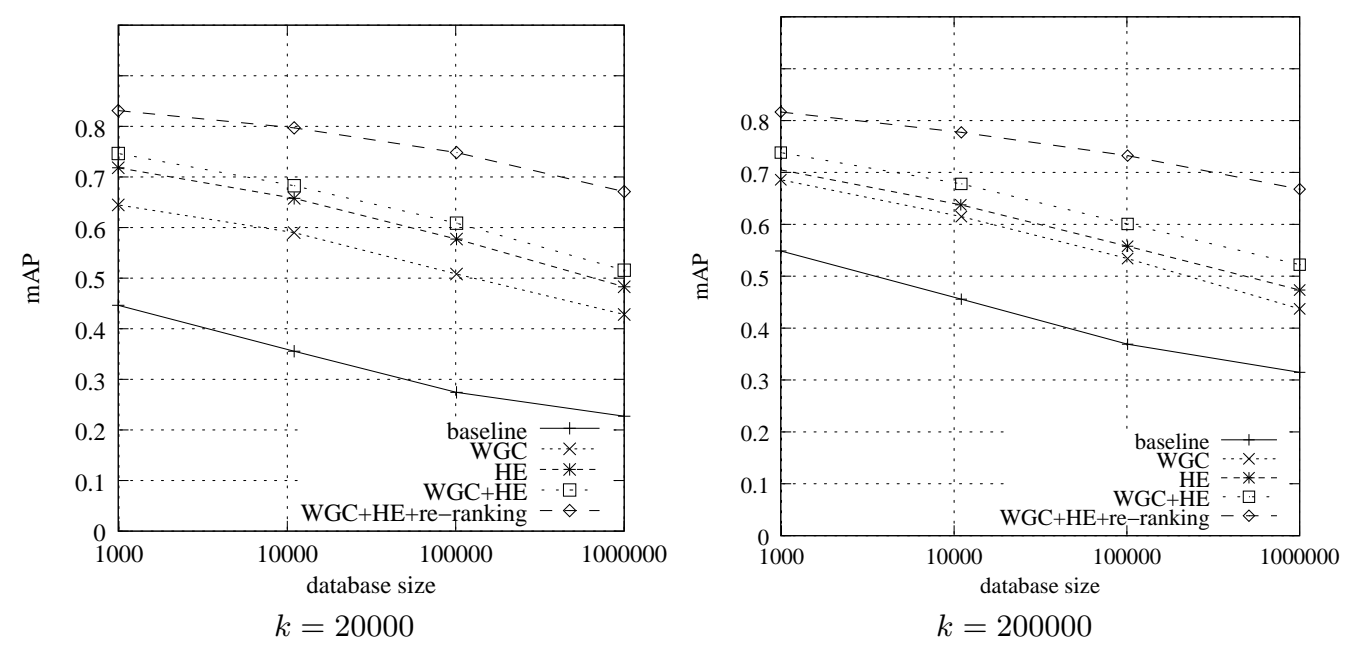

Fig. 4. Performance of the image search as a function of the dataset size for BOF, WGC, HE $\left(h_{t}=22\right)$, WGC+HE, and WGC+HE+re-ranking with a full geometrical verification (shortlist of 100 images). The dataset is Holidays with a varying number of distractors from Flickr1M.

a shortlist of 100 images. We can observe further improvement, which confirms the complementary of this step with WGC.

Table 3. Holidays dataset + Flickr1M: Rate of true positives returned in the top 100 positions as a function of the dataset size for a shortlist of 100 images, $k=200000$.

\begin{tabular}{|c|crrr|}
\hline dataset size & 1490 & 11490 & 101490 & 1001490 \\
\hline BOF & 0.673 & 0.557 & 0.431 & 0.306 \\
WGC+HE & 0.855 & 0.789 & 0.708 & 0.618 \\
\hline
\end{tabular}

\section{Conclusion}

This paper has introduced two ways of improving a standard bag-of-features representation. The first one is based on a Hamming embedding which provides binary signatures that refine visual words. It results in a similarity measure for descriptors assigned to the same visual word. The second is a method that enforces weak geometric consistency constraints and uses a priori knowledge on the geometrical transformation. These constraints are integrated within the inverted file and are used for all the dataset images. Both these methods improve the performance significantly, especially for large datasets. Interestingly, our modifications do not result in an increase of the runtime. 


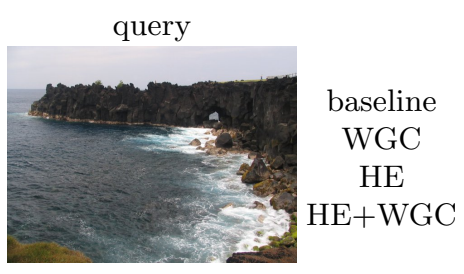

re-ranked correct results and their ranks

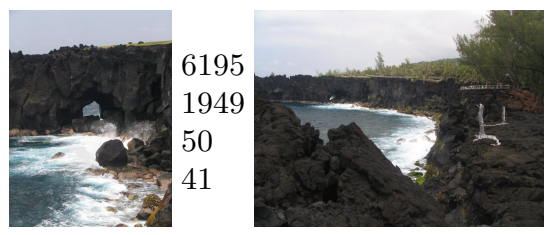

49563

7114

30657

22036

1

22036
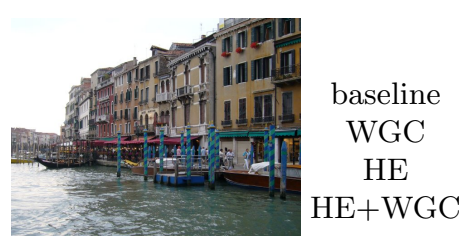

re-ranked
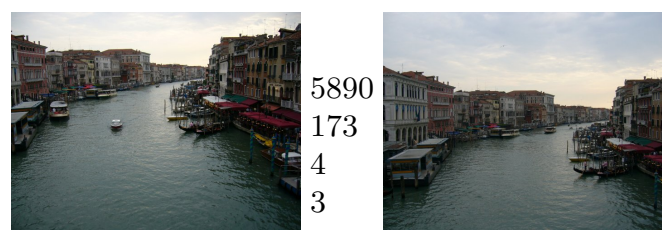

43064

1234

55

13

2

7

Fig. 5. Queries from the Holidays dataset and some corresponding results for Holidays+1M distractors Flickr1M.

Acknowledgments. We would like to acknowledge the ANR projects GAIA and RAFFUT as well as GRAVIT for their financial support.

\section{References}

1. Lowe, D.: Distinctive image features from scale-invariant keypoints. IJCV 60 (2004) 91-110

2. Mikolajczyk, K., Schmid, C.: Scale and affine invariant interest point detectors. IJCV 60 (2004) 63-86

3. Matas, J., Chum, O., Martin, U., Pajdla, T.: Robust wide baseline stereo from maximally stable extremal regions. In: BMVC. (2002) 384-393

4. Sivic, J., Zisserman, A.: Video Google: A text retrieval approach to object matching in videos. In: ICCV. (2003) 1470-1477

5. Nistér, D., Stewénius, H.: Scalable recognition with a vocabulary tree. In: CVPR. (2006) 2161-2168

6. Omercevic, D., Drbohlav, O., Leonardis, A.: High-dimensional feature matching: employing the concept of meaningful nearest neighbors. In: ICCV. (2007)

7. Datar, M., Immorlica, N., Indyk, P., Mirrokni, V.: Locality-sensitive hashing scheme based on p-stable distributions. (2004) 253-262

8. Shakhnarovich, G., Darrell, T., Indyk, P.: 3. In: Nearest-Neighbor Methods in Learning and Vision: Theory and Practice. MIT Press (2006)

9. Philbin, J., Chum, O., Isard, M., A., J.S., Zisserman: Object retrieval with large vocabularies and fast spatial matching. In: CVPR. (2007) 
10. Jegou, H., Harzallah, H., Schmid, C.: A contextual dissimilarity measure for accurate and efficient image search. In: CVPR. (2007)

11. Fraundorfer, F., Stewenius, H., Nister, D.: A binning scheme for fast hard drive based image search. In: CVPR. (2007)

12. Schindler, G., Brown, M., Szeliski, R.: City-scale location recognition. In: CVPR. (2007)

13. Philbin, J., Chum, O., Isard, M., Sivic, J., Zisserman, A.: Lost in quantization: Improving particular object retrieval in large scale image databases. In: CVPR. (2008)

14. Jegou, H., Douze, M.: INRIA Holidays dataset http://lear.inrialpes.fr/people/jegou/data.php (2008)

15. Mikolajczyk, K.: Binaries for affine covariant region descriptors. http://www.robots.ox.ac.uk/ vgg/research/affine/ (2007) 